\title{
Patterns of Symptoms and Functional Impairments in Children with Cancer
}

\author{
Tyler W. Buckner, MD ${ }^{1},{ }^{*}$, Jichuan Wang, $\mathrm{PhD}^{2,3}$, Darren A. DeWalt, MD, MPH ${ }^{1}$, Shana \\ Jacobs, MD ${ }^{2,3}$, Bryce B. Reeve, PhD ${ }^{1}$, and Pamela S. Hinds, PhD, RN, FAAN ${ }^{2,3}$ \\ ${ }^{1}$ University of North Carolina at Chapel Hill, Chapel Hill, North Carolina \\ ${ }^{2}$ Children's National Health System, Washington, District of Columbia \\ ${ }^{3}$ The George Washington University, Washington, District of Columbia
}

\begin{abstract}
Background-Children with cancer experience multiple symptoms due to their disease and as a result of treatment. The purpose of this study was to demonstrate the feasibility and potential utility of using latent profile analysis (LPA), a type of cluster analysis, in children with cancer to identify groups of patients who experience similar levels of symptom severity and impairment of physical function.
\end{abstract}

Procedure-We analyzed patient-reported symptom and functional data previously collected using the Pediatric Patient Reported Outcomes Measurement Information System (PROMIS). LPA was used to identify and characterize groups of patients who reported similar levels of symptom severity and functional impairment. We then used the multinomial logit model to examine demographic and disease characteristics associated with symptom/ function profile membership.

Results-The analysis included 200 patients in treatment or in survivorship. We identified four symptom/ function profiles; children currently receiving cancer treatment and those with at least one other medical problem were more likely to be members of the profile with the highest levels of symptom severity and functional impairment. Gender, age, race/ethnicity, and tumor type were not associated with profile membership.

Conclusions-LPA is a cluster research methodology that provides clinically useful results in pediatric oncology patients. Future studies of children with cancer using LPA could potentially lead to development of clinical scoring systems that predict patients' risk of developing more severe symptoms and functional impairments, allowing clinicians, patients, and parents to better anticipate and prevent the multiple symptoms that occur during and after treatment for childhood cancer.

() 2014 Wiley Periodicals, Inc.

"Correspondence to: Tyler W. Buckner, Physicians' Office Building, CB \# 7305, 170 Manning Drive, Chapel Hill, NC 27599-7236. tyler_buckner@med.unc.edu.

Conflict of interest: Nothing to declare. 


\section{Keywords}

cluster analysis; latent profile analysis; multiple symptoms; patient-reported outcomes; pediatric oncology

\section{INTRODUCTION}

More than 12,000 children in the United States will be diagnosed with cancer in 2014, and nearly all of them will experience multiple symptoms and functional impairments as a result of their disease and its treatment [1,2]. As we increase awareness of how these negative experiences impact children's well-being, efforts to better understand, prevent, and manage the adverse effects of cancer therapies emerge. Use of patient-reported measures of symptoms and function enhances more traditional outcome data collected in clinical studies of cancer therapy by describing each patient's personal experience with his or her illness $[3,4]$. Interpreting this valuable information can be challenging, because children with cancer experience varying degrees of symptom and functional impairments, both of which can occur alone or concurrently [5]. In this study, we applied latent profile analysis (LPA) to identify groups of children who experienced similar and dissimilar levels or patterns of cooccurring symptoms and functional impairments [6]. Using LPA to recognize which children are likely to experience these patterns of symptoms and functional impairments may help clinicians anticipate and mediate their effects on the children's quality of life.

The study of multiple symptoms, collectively termed symptom cluster research, has been used by several scientific disciplines to identify co-occurring events, behaviors, or psychological phenomena [7]. These techniques were first applied in the field of cancer outcomes research a little over a decade ago, in an effort to identify associations among symptoms experienced during cancer treatment and expand our understanding of the mechanisms underlying symptom development [8]. Researchers in this relatively nascent area of investigation have yet to reach consensus on the ideal methodology(ies) to use for the purposes of informing clinical oncology practice [9]. Most studies have utilized statistical approaches such as simple correlation, principal component analysis, common factor analysis, or cluster analysis to identify symptom groupings or clusters, such as nausea-vomiting, pain-insomnia-fatigue, and fatigue-depression [10-12]. Only a few of these investigations have involved reports from children with cancer [13-17].

LPA has not previously been used to study children with cancer, and it differs from other statistical approaches used in symptom cluster research in several important ways. LPA, one of the mixture models, is a model-based approach used to group patients into distinctive profiles. Unlike the traditional cluster analysis that uses ad hoc dissimilarity measures, such as Euclidean distance, to identify clusters, LPA identifies groups based on probability. In addition, LPA provides statistical tests and indices for model goodness-of-fit assessment $[18,19]$. Unlike factor analysis that identifies groups of variables measuring symptoms, LPA categorizes patients into groups based on their responses to the items measuring symptom severity. The goal of grouping patients into these latent profiles is to identify shared underlying characteristics that might contribute to the likelihood of membership within a 
particular latent profile [10,20,21]. For example, patients who receive a particular treatment regimen may be more likely to be members of a latent profile that experiences higher levels of symptom severity than those who receive a different treatment regimen. One potential application of these findings is the development of risk prediction tools that could be used to identify patients at the outset of cancer treatment who are more likely to become members of a particular symptom profile; foreknowledge of this risk could then be used to modify strategies designed to mitigate or prevent future symptoms. For instance, patients predicted to be at particular risk for developing higher levels of pain, depression, and anxiety might benefit from establishing a relationship with a mental health professional before these symptoms develop.

The clinical utility of symptom cluster research in pediatric oncology remains unclear [22]. The primary goal of this study is to demonstrate the feasibility and potential utility of LPA in pediatric cancer research. We also aim to demonstrate a new method for integrating measures of function into the study of patient-reported outcomes in pediatric cancer. The rationale for this approach is that our understanding of the impact of cancer and its treatment would be enriched by including both symptoms and function in analyses, because both types of outcomes help to define a patient's illness experience. Patient clustering based on symptoms and functional impairment has not previously been examined in the pediatric oncology population [23]. Providing a better understanding of the patient's responses to disease and treatments may produce a more clinically useful model, one that could potentially be applied directly to patients in the pediatric oncology clinic. The findings of this study will inform future efforts to apply symptom cluster research methodology in clinical pediatric oncology research, with the ultimate goal of improving our ability to care for children with cancer.

\section{METHODS}

\section{Participants and Data Collection}

The data used in this analysis were collected as part of the Patient-Reported Outcomes Measurement Information System ${ }^{\circledR}$ (PROMIS ${ }^{\circledR}$ ) pediatric initiative, which has been previously described [24]. Children with cancer between the ages of 8 and 17 years from 5 participating institutions provided information either while receiving treatment for cancer or after completing therapy (i.e., on-therapy or in survivorship). A total of 203 pediatric oncology patients enrolled in the study; 3 patients did not complete any PROMIS pediatric items and are therefore not included in the analysis. Patients were considered to be currently receiving cancer treatment if they had received disease-directed therapy within the previous 45 days. Participants' guardians completed questions related to patient demographics and other health problems, and each guardian was asked to provide his or her highest achieved educational level.

\section{Measures}

We included four PROMIS symptom domains (anxiety, depression, fatigue, and pain interference) and three functional domains (peer relationships, physical functioning-upper extremity, and physical functioning-mobility) in this analysis. Scores on the PROMIS 
measures are on a T-score metric, normed to have a mean of 50 and a standard deviation of 10 in the original Pediatric PROMIS patient sample [24-28]. Higher scores in the symptom domains represent greater symptom burden; in contrast, higher scores in the functional domains represent better functioning.

\section{Statistical Analyses}

We used LPA [29-34], a posterior membership probability model, to identify subgroups (profiles) of patients comprised of individuals with similar levels of symptom severity and functional impairment. The optimal number of profiles was determined by generating a series of LPA models with an increasing number of latent profiles and iteratively comparing each successive model $\mathrm{k}$ with the previous $(\mathrm{k}-1)$ model using Akaike, Bayesian, and sample-size adjusted Bayesian information criterion indices (AIC, BIC, and SABIC), the Lo-Mendell-Rubin likelihood ratio (LMR LR) test [35], the adjusted LMR LR (ALMR LR) test, and the bootstrap likelihood ratio test (BLRT) $[31,36]$.

We classified patients into their most likely latent profiles using the estimated posterior membership probabilities for each observation. The quality of membership classification was assessed by examining average posterior probabilities and the entropy statistic. Next, the prevalence rates (i.e., unconditional probabilities) of the latent profiles and the mean scores of the PROMIS measures in each latent profile were assessed. Finally, the relationships of the latent profile membership with treatment status, as well as individual patient characteristics (sex, age, race/ ethnicity, other health problems, guardian's highest education, and cancer type) were tested using a multinomial logit model, in which profile 4 was treated as the reference group. The statistical package Mplus [37] was used for modeling.

\section{RESULTS}

Demographics and clinical characteristics of the patient sample were described previously and are summarized in Table I [24]. Most patients had acute leukemia or lymphoma ( $\mathrm{n}=$ $120,60.0 \%)$; the remainder were diagnosed with solid tumors $(\mathrm{n}=58,29.0 \%)$ or brain tumors $(\mathrm{n}=22,11.0 \%)$. The patient sample was racially diverse, with 101 patients $(50.5 \%)$ reporting non-white race. The means and standard deviations of the seven PROMIS pediatric outcome measures are shown in Table II. Mean domain scores range from 47.2 to 48.9, indicating that, on average, our cancer patients are relatively similar to the reference group used during development of the pediatric PROMIS measures.

Comparison of LPA models with information criterion indices and likelihood ratio tests indicate that the data best fit either a four-or five-profile model, as the BIC, LMR LR, and ALMR LR favored four profiles and the AIC, ABIC, and BLRT favored five profiles (Table III). We selected the four-profile model for further analysis because it provided better profile membership classification and the most useful and interpretable information from a clinical perspective. Posterior assignment probabilities for Profiles 1, 2, 3, and 4 were 0.92, 0.91, 0.91 , and 0.97, respectively (i.e., the diagonal figures in Table IV), which are much higher than the standard cutoff point of 0.70 [38]. In addition, the entropy statistic of 0.88 was also 
large, indicating good membership classification [39]. Profile 1 contained 16\% of the total number of patients; profile 2, 24\%; profile 3, $16 \%$; and profile $4,45 \%$.

Each profile's estimated mean symptom and functioning scores are shown in Figure 1. The values of the PROMIS pediatric outcome measures for each profile were distinctive and showed clear patterns across the latent profiles. Patients in profile 1 had on average higher symptom severity and lower functioning than patients in any other profile. Conversely, patients in profile 4 had on average lower symptom severity and better functioning than patients in other profiles. Domain scores for profiles 2 and 3 fell between profiles 1 and 4 and presented a mixed picture: more severe symptoms but better functioning in profile 2 , and less severe symptoms but worse functioning in profile 3 .

Treatment status was the strongest predictor of profile membership (and therefore symptom burden and functioning) identified by our analysis (Table V). Children currently receiving treatment for cancer had higher symptom severity and poorer functioning compared to those who had already completed therapy, as indicated by their having larger odds of being in profiles 1, 2, or 3 compared to profile 4 ( $\mathrm{OR}=6.5$ (95\% CI: 2.4-17.3), 2.5 (1.1-5.6), and 7.1 (2.6-19.4), respectively). Likewise, children identified as having at least one other health problem in addition to cancer had higher symptom severity and worse functioning than children with no additional health problems. The corresponding odds ratios and $95 \%$ confidence intervals for membership in profiles 1,2 , and 3 were $\mathrm{OR}=6.1$ (2.3-16.0), 2.4 (1.1-5.6), and 3.6 (1.3-9.7), respectively. Gender, age, race/ethnicity, guardian education, and tumor type were not statistically significant predictors of profile membership.

\section{DISCUSSION}

Patient-reported outcomes (PROs) play an important role in helping us understand the impact of cancer and its treatments on patients' lives. Our findings suggest that among children with a variety of cancers, there are subgroups of patients who have distinct symptom- and function-related phenotypes. Patients actively receiving cancer treatment and those who have co-morbid health conditions were more likely to be classified in profiles with more severe symptoms and worse functioning than those who have completed therapy and have no additional health problems. These results are consistent with our clinical expectations and speak to the validity of LPA to summarize patterns of symptom burden and functional impairment in this population. The lack of correlation between profile membership and demographic characteristics of patients underscores the difficulty of predicting symptoms and functional impairments based solely on age, sex, and race/ ethnicity. In addition, the excellent latent profile membership classification by the model provides confirmation that the continuous variables produced by the pediatric PROMIS measures are suitable for use in LPA modeling.

Direct head-to-head comparison of the different statistical approaches used in symptom cluster research has not yet been performed. Such studies would inform future efforts to analyze symptom data by allowing researchers to select analytic strategies that yield the most clinically useful and interpretable information. Prospective evaluation of multiple symptom models to determine which approach is most predictive of change over time would 
also be informative. These investigations would potentially improve our ability to match data analysis methods with the goals of future studies.

The majority of studies of multiple symptoms in cancer patients focus on identifying groups of symptoms rather than groups of patients with similar symptom experience phenotypes. LPA, a technique for grouping patients in this way, has not previously been applied to PRO data in the pediatric cancer population [10]. As shown by our study, LPA can identify different patient phenotypes based on symptom severity and functional impairment. This approach yields results that are more specific to the experiences of individual patients, as compared to an analysis that produces findings at the level of symptoms for the total group. Identification and characterization of PRO profiles provide important insight into the realities faced by individual patients, a critical first step toward developing tools to predict and manage symptoms and functional impairments more effectively.

The concept of grouping patients into different symptom experience phenotypes was previously demonstrated by two studies in adults with cancer [40,41]. Both studies investigated the "sickness behavior" symptom cluster of pain, fatigue, sleep disturbance, and depression in adult outpatients with cancer diagnoses. The investigators used statistical techniques similar to those employed by our study to identify distinct groups of patients, each with a different profile of symptom severity. Notably, the proportions of patients who fell into the "all high severity" and "all low severity" groups in these studies were similar to the proportions of patients in our study in Profiles 1 (all high severity) and 4 (all low severity).

In contrast to our study, most cluster research studies in pediatric and adult oncology do not include measures of function in their analyses [5,9]. Including both symptoms and functional outcomes when assessing patients' responses to illness is essential to our understanding how cancer affects patient quality of life. Previous studies have demonstrated that functional status not only affects quality of life directly, but also partially mediates the effects of symptoms on quality of life [42]. These complex interactions cannot be fully appreciated and studied using cluster research methods that do not include measures of function as outcomes.

The discordance between symptom severity and functional status seen in profiles 2 and 3 in our study highlights the potential utility of including measures of function in LPA profiles. This unexpected result raises several interesting questions about what unmeasured factors might contribute to the likelihood of membership in these profiles, and it suggests that the interactions among symptoms and functional status are more complex than we would otherwise appreciate had function not been included in our analysis. In contrast to our study, the adult cancer studies described above evaluated the impact of symptom profile membership on physical function, rather than including measures of function in the profiles. The studies found that the groups of patients who experienced higher levels of symptoms had significantly lower functional status, as measured by the Karnofsky Performance Score $[40,41]$. This finding was expected, and it aligns with the levels of symptom severity and function observed in profiles 1 and 4 in our study (Fig. 1). Profiles 2 and 3, however, revealed the unexpected finding that symptom severity and functional outcomes may not be 
inextricably linked — a finding that needs to be confirmed and explored by additional investigations in this population. In this way, our study demonstrates that including both symptoms and functional outcomes in cluster analysis provides a better picture of the impact of cancer and its treatment on children's well-being.

The results of our study suggest that it may be possible to use LPA to create a useful tool for clinical pediatric oncology practice. Identification of symptom/function profiles and the factors associated with profile membership could be used to develop a clinical prediction score that prospectively evaluates a patient's risk for developing varying degrees of symptom severity and functional impairment during the course of cancer treatment and follow up. Making the patterns identified through LPA available to clinicians would potentially improve their ability to provide more tailored information for families to help them to better anticipate how their child could respond to the cancer treatment experience. Foreknowledge of this risk might allow clinicians to modify strategies aimed at preventing or mitigating negative outcomes. This is an important goal, as the link between a child's symptom experience during cancer treatment and his or her quality of life is clear [43,44]. Factors that influence health-related quality of life during cancer treatment may also have an impact on childhood development; studying how symptom and functional profiles change over time and how they differ by age of the patient might also shed light on how cancer treatment affects long-term outcomes and late effects of therapy.

In addition to developing predictive models of profile membership, LPA can also be used to investigate biologic mechanisms that underlie specific groups of symptoms. Given the observed lack of correlation between symptom profiles and most demographic, disease, and treatment-related factors seen in previous studies, investigators hypothesized that there may be genetic variations contributing to patients' experiences with cancer [40,41]. This observation led to a study of adult oncology patients that used LPA to identify subgroups of patients with different severities of the "sickness behavior" symptom cluster (as described above) [45]. The identified subgroups were then correlated with polymorphisms in candidate genes believed to contribute to the symptoms of the sickness behavior cluster. The investigators identified a single nucleotide polymorphism in the interleukin-4 gene that was associated with high levels of all four symptoms, suggesting a possible link between this variant of IL-4 and an individual's susceptibility to experiencing more severe symptoms during cancer treatment [45]. Others have previously proposed such a connection, but this finding awaits further confirmation [46].

Our study raises important questions that are not answered by our analysis due to several limitations. The low number of patients with specific cancer types (most notably brain tumor) prevented us from determining whether the patient profiles were invariable across individual disease types. The odds ratios provided by our model would likely have been more precise (i.e., more narrow confidence intervals) with a larger number of patients. Limited clinical data were available in our study, making it impossible to examine in more detail patient and disease characteristics that might affect profile membership. In addition, the setting and timing of the patient assessments were not standardized, so the effects of these differences in questionnaire administration on profile membership are not known. Finally, the measures used in this analysis were collected at a single point in time for each 
participant, so we were unable to evaluate how profile characteristics might change over time. For example, we cannot determine whether levels of symptom severity and functional impairment change during periods of more intensive versus less intensive treatment, and we do not know whether patients shift from one profile to another during the course of treatment and survivorship. Longitudinal studies of symptoms and functional impairments using latent transition analysis, a technique similar to LPA, would provide insight into whether the observed profiles persist over time $[47,48]$.

The ultimate aim of this field of research is to improve the well-being of patients with cancer through better prevention, recognition, and management of patients' symptoms and functional impairments. The results of our study suggest that future investigations using LPA could be used to generate useful clinical tools, such as a clinical prediction score, complementing the work already underway using LPA to identify genetic variations that affect patients' experiences with cancer. In these ways, LPA research offers many new opportunities to better understand the experience of being treated for cancer as a child. The conclusions drawn from this exploratory study of secondary data should not necessarily be accepted at face value, but they should be viewed as strong evidence of the potential utility of this type of analysis. Future studies of PROs in pediatric cancer patients should include detailed plans to evaluate a broader set of symptoms, functional status, and overall quality of life domains, so that we can glean as much knowledge as possible from these efforts to improve the care of children with cancer.

\section{ACKNOWLEDGEMENTS}

Darren DeWalt and Bryce Reeve are unpaid members of the board for the PROMIS Health Organization which holds the copyright for the PROMIS instruments used in this study. We are grateful to these individuals who made meaningful contributions to this work: Suzanne L. Nuss, PhD, RN (Director, Care Transitions and Nursing Outcomes, Nebraska Medical Center), Janice S. Withycombe, PhD, RN, MN, CCRP (Research Nurse, Palmetto Health), and Kathleen Ruccione, PhD, MPH, RN, CPON, FAAN (Co-Director, HOPE Program, Children's Center for Cancer and Blood Diseases, Children's Hospital Los Angeles). This analysis was supported in part by a National Research Service Award Post-Doctoral Traineeship from the Agency for Healthcare Research and Quality sponsored by the Cecil G. Sheps Center for Health Services Research, UNC-Chapel Hill, Grant No. 5T32HS000032. PROMIS $®$ was funded with cooperative agreements from the National Institutes of Health (NIH) Common Fund Initiative (Northwestern University, PI: David Cella, PhD, U54AR057951, U01AR052177; Northwestern University, PI: Richard C. Gershon, PhD, U54AR057943; American Institutes for Research, PI: Susan (San) D. Keller, PhD, U54AR057926; State University of New York, Stony Brook, PIs: Joan E. Broderick, $\mathrm{PhD}$ and Arthur A. Stone, PhD, U01AR057948, U01AR052170; University of Washington, Seattle, PIs: Heidi M. Crane, MD, MPH, Paul K. Crane, MD, MPH, and Donald L. Patrick, PhD, U01AR057954; University of Washington, Seattle, PI: Dagmar Amtmann, PhD, U01AR052171; University of North Carolina, Chapel Hill, PI: Harry A. Guess, MD, PhD (deceased), Darren A. DeWalt, MD, MPH, U01AR052181; Children's Hospital of Philadelphia, PI: Christopher B. Forrest, MD, PhD, U01AR057956; Stanford University, PI: James F. Fries, MD, U01AR052158; Boston University, PIs: Alan Jette, PT, PhD, Stephen M. Haley, PhD (deceased), and David Scott Tulsky, PhD (University of Michigan, Ann Arbor), U01AR057929; University of California, Los Angeles, PIs: Dinesh Khanna, MD (University of Michigan, Ann Arbor) and Brennan Spiegel, MD, MSHS, U01AR057936; University of Pittsburgh, PI: Paul A. Pilkonis, PhD, U01AR052155; Georgetown University, PIs: Carol. M. Moinpour, PhD (Fred Hutchinson Cancer Research Center, Seattle) and Arnold L. Potosky, PhD, U01AR057971; Children's Hospital Medical Center, Cincinnati, PI: Esi M. Morgan DeWitt, MD, MSCE, U01AR057940; University of Maryland, Baltimore, PI: Lisa M. Shulman, MD, U01AR057967; and Duke University, PI: Kevin P. Weinfurt, PhD, U01AR052186). NIH Science Officers on this project have included Deborah Ader, PhD, Vanessa Ameen, MD (deceased), Susan Czajkowski, PhD, Basil Eldadah, MD, PhD, Lawrence Fine, MD, DrPH, Lawrence Fox, MD, PhD, Lynne Haverkos, MD, MPH, Thomas Hilton, PhD, Laura Lee Johnson, PhD, Michael Kozak, PhD, Peter Lyster, PhD, Donald Mattison, MD, Claudia Moy, PhD, Louis Quatrano, PhD, Bryce Reeve, PhD, William Riley, PhD, Peter Scheidt, MD, Ashley Wilder Smith, PhD, MPH, Susana Serrate-Sztein, MD, William Phillip Tonkins, DrPH, Ellen Werner, PhD, Tisha Wiley, PhD, and James Witter, MD, PhD. The contents of this article uses data developed under PROMIS. These contents do not necessarily represent an endorsement by the US Federal Government or PROMIS. See www.nihpromis.org for additional information on the PROMIS $®$ initiative. 


\section{REFERENCES}

1. Robison LL, Armstrong GT, Boice JD, et al. The Childhood Cancer Survivor Study: A National Cancer Institute-supported resource for outcome and intervention research. J Clin Oncol. 2009; 27:2308-2318. [PubMed: 19364948]

2. National Cancer Institute UDoHaHS. National Cancer Institute. [March 6] A Snapshot of Pediatric Cancers. 2013. http://www.cancer.gov/researchandfunding/snapshots/pdf/Pediatric-Snapshot.pdf. Published October, 2012

3. Barsevick AM. The elusive concept of the symptom cluster. Oncol Nurs Forum. 2007; 34:971-980. [PubMed: 17878126]

4. Dodd MJ, Miaskowski C, Lee KA. Occurrence of symptom clusters. J Natl Cancer Inst Monogr. 2004; (32):76-78. [PubMed: 15263044]

5. Baggott C, Dodd M, Kennedy C, et al. Multiple symptoms in pediatric oncology patients: A systematic review. J Pediatr Oncol Nurs. 2009; 26:325-339. [PubMed: 19687466]

6. Aktas A. Cancer symptom clusters: Current concepts and controversies. Curr Opin Support Palliat Care. 2013; 7:38-44. [PubMed: 23287418]

7. Barsevick AM, Whitmer K, Nail LM, et al. Symptom cluster research: Conceptual, design, measurement, and analysis issues. J Pain Symptom Manage. 2006; 31:85-95. [PubMed: 16442485]

8. Dodd MJ, Miaskowski C, Paul SM. Symptom clusters and their effect on the functional status of patients with cancer. Oncol Nurs Forum. 2001; 28:465-470. [PubMed: 11338755]

9. Xiao C. The state of science in the study of cancer symptom clusters. Eur J Oncol Nurs. 2010; 14:417-434. [PubMed: 20599421]

10. Kim HJ, Abraham I, Malone PS. Analytical methods and issues for symptom cluster research in oncology. Curr Opin Support Palliat Care. 2013; 7:45-53. [PubMed: 23196378]

11. Kirkova J, Aktas A, Walsh D, et al. Cancer symptom clusters: Clinical and research methodology. J Palliat Med. 2011; 14:1149-1166. [PubMed: 21861613]

12. Kim HJ, Abraham IL. Statistical approaches to modeling symptom clusters in cancer patients. Cancer Nurs. 2008; 31:E1-E10. [PubMed: 18772651]

13. Yeh CH, Chiang YC, Chien LC, et al. Symptom clustering in older Taiwanese children with cancer. Oncol Nurs Forum. 2008; 35:273-281. [PubMed: 18321840]

14. Hockenberry MJ, Hooke MC, Gregurich M, et al. Symptom clusters in children and adolescents receiving cisplatin, doxorubicin, or ifosfamide. Oncol Nurs Forum. 2010; 37:E16-E27. [PubMed: 20044328]

15. Hockenberry MJ, Hooke MC, McCarthy K, et al. Sickness behavior clustering in children with cancer. J Pediatr Oncol Nurs. 2011; 28:263-272. [PubMed: 21946193]

16. Baggott C, Cooper BA, Marina N, et al. Symptom cluster analyses based on symptom occurrence and severity ratings among pediatric oncology patients during myelosuppressive chemotherapy. Cancer Nurs. 2012; 35:19-28. [PubMed: 21921793]

17. Atay S, Conk Z, Bahar Z. Identifying symptom clusters in paediatric cancer patients using the Memorial Symptom Assessment Scale. Eur J Cancer Care (Engl). 2012; 21:460-468. [PubMed: 22335457]

18. Wang J, Lanza ST. Preface of methods and applications of mixture models, special journal issue of advances and applications of statistical sciences. Adv Appl Stat Sci. 2010; 3:1-6.

19. Wang, J.; Wang, X. Structural equation modeling with Mplus: Methods and applications. Wiley; Hoboken, NJ: 2012. p. 478

20. Barsevick AM, Aktas A. Cancer symptom cluster research: New perspectives and tools. Curr Opin Support Palliat Care. 2013; 7:36-37. [PubMed: 23314017]

21. Miaskowski C, Aouizerat BE, Dodd M, et al. Conceptual issues in symptom clusters research and their implications for quality-of-life assessment in patients with cancer. J Natl Cancer Inst Monogr. 2007; (37):39-46. [PubMed: 17951230]

22. Barsevick AM. The concept of symptom cluster. Semin Oncol Nurs. 2007; 23:89-98. [PubMed: 17512435] 
23. Rodgers CC, Hooke MC, Hockenberry MJ. Symptom clusters in children. Curr Opin Support Palliat Care. 2013; 7:67-72. [PubMed: 23108342]

24. Hinds PS, Nuss SL, Ruccione KS, et al. PROMIS pediatric measures in pediatric oncology: Valid and clinically feasible indicators of patient-reported outcomes. Pediatr Blood Cancer. 2013; 60:402-408. [PubMed: 22829446]

25. Irwin DE, Stucky BD, Langer MM, et al. PROMIS Pediatric Anger Scale: An item response theory analysis. Qual Life Res. 2012; 21:697-706. [PubMed: 21785833]

26. Irwin DE, Stucky B, Langer MM, et al. An item response analysis of the pediatric PROMIS anxiety and depressive symptoms scales. Qual Life Res. 2010; 19:595-607. [PubMed: 20213516]

27. Varni JW, Stucky BD, Thissen D, et al. PROMIS Pediatric Pain Interference Scale: An item response theory analysis of the pediatric pain item bank. J Pain. 2010; 11:1109-1119. [PubMed: 20627819]

28. DeWitt EM, Stucky BD, Thissen D, et al. Construction of the eight-item patient-reported outcomes measurement information system pediatric physical function scales: Built using item response theory. J Clin Epidemiol. 2011; 64:794-804. [PubMed: 21292444]

29. Arminger, G.; Clogg, CC.; Sobel, ME. Handbook of statistical modeling for the social and behavioral sciences. Plenum Press; New York: 1995. p. 592

30. Collins, LM.; Lanza, ST. Latent class and latent transition analysis: With applications in the social behavioral, and health sciences. Wiley; Hoboken, NJ: 2010. p. 285

31. McCutcheon, AL. Latent class analysis. Sage Publications; Newbury Park: 1987. p. 96

32. Marcoulides, GA.; Schumacker, RE. New developments and techniques in structural equation modeling. Lawrence Erlbaum Associates; Mahwah, NJ: 2001. p. 333

33. Muthen BO, Beyond SEM. General latent variable modeling. Behaviormetrika. 2002; 29:81-117.

34. Kaplan, D.; Sage Publications, Inc.. The Sage handbook of quantitative methodology for the social sciences. Sage; Thousand Oaks, CA: 2004. p. 511

35. Lo Y, Mendell NR, Rubin DB. Testing the number of components in a normal mixture. Biometrika. 2001; 88:767-778.

36. McLachlan, GJ.; Peel, D. Finite mixture models. Wiley; New York: 2000. p. xxiip. 419

37. Muthen, L.; Muthen, B. Mplus user's guide. 6th edition.. Muthen \& Muthen; Los Angeles, CA: 1998-2010. p. 752

38. Nagin, DS. Group-based modeling of development. Harvard University Press; London, UK: 2005. p. 214

39. Clark, SL. Mixture modeling with behavioral data. Doctoral dissertation. University of California; Los Angeles: 2010.

40. Miaskowski C, Cooper BA, Paul SM, et al. Subgroups of patients with cancer with different symptom experiences and quality-of-life outcomes: A cluster analysis. Oncol Nurs Forum. 2006; 33:E79-E89. [PubMed: 16955115]

41. Pud D, Ben Ami S, Cooper BA, et al. The symptom experience of oncology outpatients has a different impact on quality-of-life outcomes. J Pain Symptom Manage. 2008; 35:162-170. [PubMed: 18082357]

42. Hsu MC, Tu CH. Improving quality-of-life outcomes for patients with cancer through mediating effects of depressive symptoms and functional status: A three-path mediation model. J Clin Nurs. 2013 Epub ahead of print.

43. Tseng TH, Cleeland CS, Wang XS, et al. Assessing cancer symptoms in adolescents with cancer using the Taiwanese version of the M.D. Anderson Symptom Inventory. Cancer Nurs. 2008; 31:E9-E16. [PubMed: 18453871]

44. Ewing JE, King MT, Smith NF. Validation of modified forms of the PedsQL generic core scales and cancer module scales for adolescents and young adults (AYA) with cancer or a blood disorder. Qual Life Res. 2009; 18:231-244. [PubMed: 19165624]

45. Illi J, Miaskowski C, Cooper B, et al. Association between pro- and anti-inflammatory cytokine genes and a symptom cluster of pain, fatigue, sleep disturbance, and depression. Cytokine. 2012; 58:437-447. [PubMed: 22450224] 
46. Kim HJ, Barsevick AM, Fang CY, et al. Common biological pathways underlying the psychoneurological symptom cluster in cancer patients. Cancer Nurs. 2012; 35:E1-E20. [PubMed: 22228391]

47. Stephens RL, Petras H, Fabian A, et al. Patterns of functional impairment and their change among youth served in systems of care: An application of latent transition analysis. J Behav Health Serv Res. 2010; 37:491-507. [PubMed: 19688597]

48. Bray BC, Lanza ST, Collins LM. Modeling relations among discrete developmental processes: A general approach to associative latent transition analysis. Struct Equ Modeling. 2010; 17:541-569. [PubMed: 21572599] 


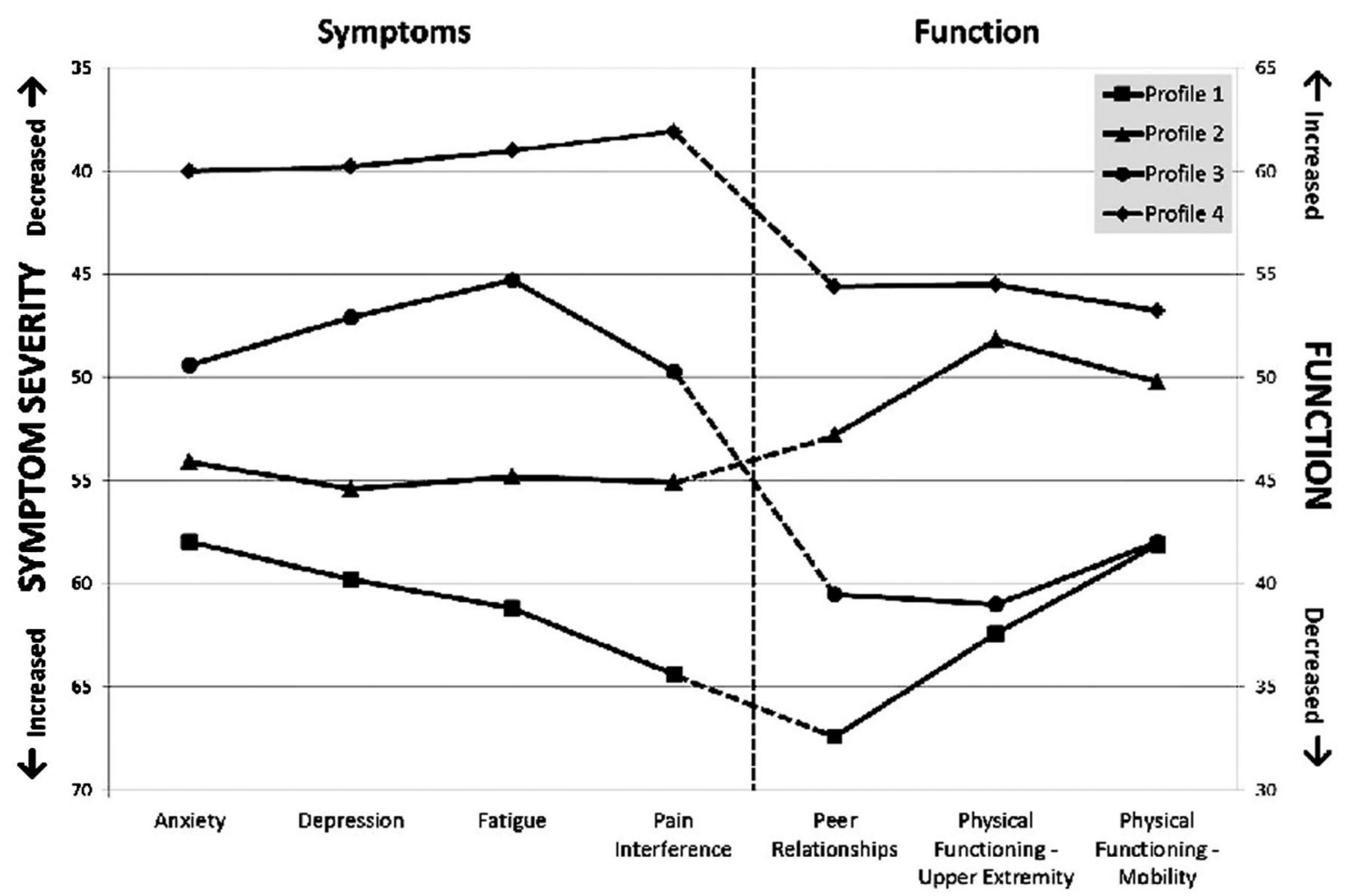

Fig. 1.

Mean PROMIS domain scores of four latent profiles generated by LPA for 200 children with cancer. Domain scores are on a T-score metric, normed in the general pediatric population to have a mean of 50 and a standard deviation of 10. PROMIS, Patient Reported Outcomes Measurement Information System; LPA, latent profile analysis. 


\section{TABLE I}

\section{Clinical and Demographic Characteristics of the Pediatric PROMIS Patient Sample}

\begin{tabular}{|c|c|}
\hline Characteristics & No. participants $(\% ; n=200)$ \\
\hline \multicolumn{2}{|l|}{ Sex } \\
\hline Male & $111(55.5)$ \\
\hline Female & $89(44.5)$ \\
\hline \multicolumn{2}{|l|}{ Age } \\
\hline $8-12$ years & $91(45.5)$ \\
\hline $13-17$ years & $109(54.5)$ \\
\hline Age (mean, SD) & $12.9(2.9)$ \\
\hline \multicolumn{2}{|l|}{ Race/ethnicity } \\
\hline Non-Hispanic white & 99 (49.5) \\
\hline Black or African American & $41(20.5)$ \\
\hline Hispanic & $40(20.0)$ \\
\hline Others & $20(10.0)$ \\
\hline \multicolumn{2}{|l|}{ Parent/Caregiver's education level } \\
\hline$\$ 8$ th grade & $4(2.0)$ \\
\hline Some high school & $5(2.5)$ \\
\hline High school degree/GED & $42(21.0)$ \\
\hline Some college/technical degree & $74(37.0)$ \\
\hline College degree & $49(24.5)$ \\
\hline Advanced degree & $26(13.0)$ \\
\hline \multicolumn{2}{|l|}{ History of other health problems } \\
\hline No other health problems & $132(66.0)$ \\
\hline Yes $=1$ other health problem & $39(19.5)$ \\
\hline Yes $\geq 2$ other health problems & $29(14.5)$ \\
\hline \multicolumn{2}{|l|}{ Type of cancer } \\
\hline Leukemia/lymphoma & $120(60.0)$ \\
\hline Brain tumor & $22(11.0)$ \\
\hline Solid tumor & $58(29.0)$ \\
\hline
\end{tabular}

PROMIS, Patient Reported Outcomes Measurement Information System; SD, standard deviation; GED, General Equivalency Diploma. 


\section{TABLE II}

Symptom and Physical Function Domain Scores of the Pediatric Oncology PROMIS Patient Sample (N = $200)^{*}$

\begin{tabular}{ll}
\hline Domain & Mean (SD) \\
\hline Anxiety & $47.7(11.7)$ \\
Depression & $47.6(10.2)$ \\
Fatigue & $48.0(13.4)$ \\
Pain interference & $47.2(11.3)$ \\
Peer relationships & $48.9(10.4)$ \\
Physical functioning-upper extremity & $48.8(8.7)$ \\
Physical functioning-mobility & $47.0(10.2)$ \\
\hline
\end{tabular}

PROMIS, Patient Reported Outcomes Measurement Information System.

* Sample size slightly varies by measures due to missing values. 


\section{TABLE III}

Comparisons of Different LPA Models Using Information Criterion Indices and Likelihood Ratio Tests

\begin{tabular}{lllllll}
\hline & & & & \multicolumn{3}{c}{ P-Value } \\
\cline { 5 - 7 } Model & AIC & BIC & ABIC & LMR LR & ALMR LR & BLRT \\
\hline 1-Profile LCA & $10,294.38$ & $10,340.56$ & $10,296.20$ & - & - & - \\
2-Profile LCA & $9,859.745$ & $9,932.308$ & $9,862.610$ & $<0.0001$ & $<0.0001$ & $<0.0001$ \\
3-Profile LCA & $9,793.360$ & $9,892.309$ & $9,797.266$ & 0.0296 & 0.0318 & $<0.0001$ \\
4-Profile LCA & $9,723.661$ & $9,848.997$ & $9,728.609$ & 0.0148 & 0.0164 & $<0.0001$ \\
5-Profile LCA & $9,709.378$ & $9,861.101$ & $9,715.368$ & 0.2057 & 0.2153 & $<0.0001$ \\
\hline
\end{tabular}

Not applicable. LPA, latent profile analysis; AIC, Akaike information criterion; BIC, Bayesian information criterion; ABIC, sample-size adjusted Bayesian information criterion; LMR LR, Lo-Mendell-Rubin likelihood ratio test; ALMR LR, adjusted Lo-Mendell-Rubin likelihood ratio test; BLRT, bootstrap likelihood ratio test. 


\section{TABLE IV}

Latent Profile Assignment Probabilities for Each Profile in the Four-Profile LPA Model

\begin{tabular}{lllll}
\hline & Profile 1 & Profile 2 & Profile 3 & Profile 4 \\
\hline Profile 1 $(\mathrm{N}=31,15.5 \%)$ & 0.92 & 0.03 & 0.04 & 0 \\
Profile 2 $(\mathrm{N}=47,23.5 \%)$ & 0.02 & 0.91 & 0.03 & 0.04 \\
Profile 3 $(\mathrm{N}=32,16.0 \%)$ & 0.05 & 0.03 & 0.91 & 0.02 \\
Profile 4 $(\mathrm{N}=90,45.0 \%)$ & 0 & 0.02 & 0.01 & 0.97 \\
& \multicolumn{5}{l}{ Entropy $=0.88$} \\
\hline
\end{tabular}

Latent profile classification is based on the most likely latent class membership. LPA, latent profile analysis. 
TABLE V

Predictors of Latent Profile Membership in the Latent Multinomial Logit Model

\begin{tabular}{|c|c|c|c|c|c|c|c|c|}
\hline \multirow[t]{3}{*}{ Covariate } & \multicolumn{8}{|c|}{ Latent profile } \\
\hline & \multicolumn{2}{|c|}{ Profile $1(n=31)$} & \multicolumn{2}{|c|}{ Profile $2(n=47)$} & \multicolumn{2}{|c|}{ Profile $3(n=32)$} & \multicolumn{2}{|c|}{ Profile $4^{a}(n=90)$} \\
\hline & Odds ratio & $95 \% \mathrm{CI}$ & Odds ratio & $95 \%$ CI & Odds ratio & $95 \% \mathrm{CI}$ & Odds ratio & $95 \% \mathrm{CI}$ \\
\hline \multicolumn{9}{|l|}{ Sex } \\
\hline Female & - & - & - & - & - & - & - & - \\
\hline Male & 0.9 & $0.3,2.2$ & 0.6 & $0.3,1.3$ & 0.5 & $0.2,1.2$ & - & - \\
\hline Age & 1.1 & $0.9,1.3$ & 1.1 & $0.9,1.2$ & 0.9 & $0.8,1.1$ & - & - \\
\hline \multicolumn{9}{|l|}{ Race/ethnicity } \\
\hline Non-Hispanic White & - & - & - & - & - & - & - & - \\
\hline Black/African American & 0.8 & $0.2,2.6$ & 2.0 & $0.8,5.1$ & 1.0 & $0.2,4.3$ & & \\
\hline Hispanic & 0.8 & $0.2,2.7$ & 1.1 & $0.4,3.3$ & 2.9 & $0.9,9.8$ & & \\
\hline Other & 0.6 & $0.1,3.6$ & 1.6 & $0.4,6.1$ & 2.9 & $0.7,12.2$ & - & - \\
\hline \multicolumn{9}{|l|}{ Other health problems } \\
\hline No other health problems & - & - & - & - & - & - & - & - \\
\hline Other health problems $\geq 1$ & $6.1^{*}$ & $2.3,16.0$ & $2.4^{*}$ & $1.1,5.6$ & $3.6^{*}$ & $1.3,9.7$ & - & - \\
\hline Guardian's highest education & 0.7 & $0.5,1.03$ & 0.8 & $0.5,1.1$ & 1.6 & $0.99,2.6$ & - & - \\
\hline \multicolumn{9}{|l|}{ Type of tumor } \\
\hline Leukemia/lymphoma & - & - & - & - & - & - & - & - \\
\hline Brain tumor & 1.0 & $0.2,5.8$ & 1.1 & $0.3,4.1$ & 2.4 & $0.6,9.2$ & & \\
\hline Solid tumor & 2.3 & $0.8,6.1$ & 1.5 & $0.7,3.6$ & 0.6 & $0.2,1.9$ & & \\
\hline \multicolumn{9}{|l|}{ Child is in active treatment } \\
\hline No & - & - & - & - & - & - & - & - \\
\hline Yes & $6.5^{*}$ & $2.4,17.3$ & $2.5^{*}$ & $1.1,5.6$ & $7.1^{*}$ & $2.6,19.4$ & - & - \\
\hline
\end{tabular}

CI, confidence interval.

${ }^{a}$ Reference group.

${ }^{*} P<0.05$. 